\title{
Are two heuristics better than one? The fluency and distinctiveness heuristics in recognition memory
}

\author{
Marianne E. Lloyd • Jeremy K. Miller
}

Published online: 6 April 2011

(C) Psychonomic Society, Inc. 2011

\begin{abstract}
Four experiments were conducted to test the impact of having multiple heuristics (distinctiveness and fluency) available during a recognition test. Recent work by Gallo, Perlmutter, Moore, and Schacter (Memory \& Cognition 36:461-466, 2008) suggested that fluency effects are reduced when the distinctiveness heuristic can be applied to a recognition decision. In Experiment 1, we used a response reversal paradigm (Van Zandt \& Maldonado-Molina Journal of Experimental Psychology: Learning, Memory, and Cognition 30:1147-1166, 2004) to demonstrate that participants transitioned from an early response strategy that was largely reliant on fluency to a later strategy in which the influences of fluency and distinctiveness were both observable. Experiments $2 \mathrm{a}, 2 \mathrm{~b}$, and 3 showed no evidence for reduction of the fluency heuristic after picture study when the test required a delayed response (Exp. 2a), confidence ratings (Exp. 2b), or the application of conceptual fluency (Exp. 3). The results are consistent with models of memory that assume that familiarity and recollection influence individual memory decisions Wixted (Psychological Review, 114:152-176, 2007).
\end{abstract}

Keywords Familiarity in recognition memory.

Recollection $\cdot$ Metamemory $\cdot$ Heuristics

\footnotetext{
M. E. Lloyd $(\square)$

Department of Psychology, Seton Hall University,

400 South Orange Avenue,

South Orange, NJ 07079, USA

e-mail: marianne.lloyd@shu.edu

J. K. Miller

Department of Psychology, Willamette University,

Salem, OR, USA
}

Recognition memory decisions are influenced by a number of automatic and strategic factors (for reviews, see Diana, Reder, Arndt \& Park, 2006; Yonelinas, 2002). In a typical recognition memory experiment, participants are asked to distinguish a list of recently studied targets from a similar set of lure items. As participants evaluate the strength of their memory for each successive test item, they may engage in a number of memory-monitoring strategies designed to help them differentiate targets from lures. One such strategy is the fluency heuristic. The fluency heuristic refers to the finding that when test items are made faster to process through either semantic (e.g., Whittlesea, 1993) or perceptual (e.g., Jacoby \& Whitehouse, 1989) priming techniques, the increase in fluency leads to a higher probability that participants will claim that a test item is old. One explanation of the link between high levels of processing fluency and increased feelings of familiarity is that the link is based on participants adjusting their memory strategies to reflect the positive correlation between previous experience and fluent processing (e.g., Jacoby \& Dallas, 1981; Murrell \& Morton, 1974; Neisser, 1954). Consequently, participants interpret fluency as a form of evidence suggesting that the stimulus has been recently experienced. Typically, fluency effects are described as leading to a subjective feeling of familiarity that leads to an increased likelihood of a positive recognition decision. However, fluent processing of a stimulus is not invariably interpreted as evidence that an item has been previously experienced. Several factors have been shown to moderate participants' strategies regarding when and where to attribute fluency as evidence of previous occurrence.

The factors that seem to moderate fluency attribution strategies can be broadly divided into two categories: those derived from cues at study and those derived from cues at test. Generally, people seem to track properties of the 
encoding episode in order to assess the degree to which the properties of that episode were consistent with the test circumstances. For example, elements of the study episode that have been shown to have an influence on subsequent fluency attribution strategies include the match between the study and test formats (Clarke \& Morton, 1983; Jacoby \& Dallas, 1981), the number exposures for each study item (Lloyd, Westerman \& Miller, 2003), and the sensory modality in which study items are presented (Westerman, Lloyd \& Miller, 2002). Encoding such factors as these plays a vital role in establishing the participant's beliefs about the relevance of fluency as a memory cue. When there is a high degree of overlap between encoding and test circumstances, participants are more likely to attribute fluency as evidence of previous experience.

Furthermore, the properties of the recognition test can influence the strategic attribution of fluency as well. For example, when participants are exposed to a recognition test during which half of the test items are primed by briefly presenting the identical word prior to the recognition test word, the properties of the prime have been shown to influence recognition performance. Varying the exposure duration of these primes has been shown to affect participants' attribution strategies. When prime durations are long (e.g., $250 \mathrm{~ms}$ ), participants are less likely to attribute fluency as evidence of previous occurrence, relative to when primes are of a shorter duration (e.g., 34 ms; Bernstein \& Welch, 1991; Jacoby \& Whitehouse, 1989). Further, the relative frequency of primed versus unprimed test items has an influence on attribution strategies, such that when primed items are infrequent on the test, participants are more likely to attribute fluency as evidence of previous occurrence (Westerman, 2008). These results demonstrate that the salience of the fluency cue and the level of fluency relative to other test items are important influences on attribution strategies in simple yes/no recognition tests (Whittlesea, Jacoby \& Girard, 1990). Generally, when people engage the fluency heuristic to make a memory decision, they strategically adjust their response strategies to account for the properties of the encoding environment (e.g., knowledge concerning the study episode, such as how frequently and clearly words were encoded) and the properties of a person's phenomenological recognition experience (how fluently a word is processed).

The fluency heuristic is one process that contributes to a person's judgment of the overall memorability of a stimulus. However, a diverse literature has suggested that memory decisions may not be made solely on the basis of familiarity. In this view, it is believed that participants utilize a second distinct memory process known as recollection in which they are able to effortfully retrieve detailed representations of an event from their pasts (Yonelinas, 1999, 2002). Critically, the process of recollection can also be governed by heuristic decision-making processes. For example, the distinctiveness heuristic is a recollection-based heuristic that participants can use to reduce their false alarm rates on a recognition test (Dodson \& Hege, 2005; Dodson \& Schacter, 2002; Schacter, Israel \& Racine, 1999). In a typical experiment examining the distinctiveness heuristic, participants study a set of words alone or the same set of words that have been made distinctive (often by presenting a picture with the word). The typical finding is that true recognition is equivalent for both groups, whereas the picture condition has a lower false alarm rate. The explanation for this finding is that participants in the picture condition use the distinctiveness heuristic to lower their false alarm rate. They come to expect distinctive information to accompany a memory for an item, and if this distinctive information is lacking, the item is rejected as a lure. The extent to which recollection-based memory strategies such as the distinctiveness heuristic may interact with familiarity-based strategies such as the fluency heuristic has received little empirical attention.

Recently, Gallo, Perlmutter, Moore and Schacter (2008) took the first steps toward unraveling the question of how fluency attribution strategies interact with other memory strategies, such as the distinctiveness heuristic. In Gallo et al.'s (2008) experiments, participants had access to both fluency and distinctiveness information as they made their recognition decisions. The researchers demonstrated that the fluency heuristic and the distinctiveness heuristic interacted in meaningful ways. Generally, it was found that distinctive encoding reduced the magnitude of the fluency illusion. That is, participants who have had a distinctive encoding episode were less likely to rely on fluency as a cue to memory as they made recognition decisions. In the experiments, participants were exposed to study lists of varying levels of distinctiveness. For some participants, each study word was followed by a corresponding picture (high-distinctiveness condition), while another group of participants saw each study word presented followed by an auditory presentation of the word. During the recognition test, half of the items were primed by presentation of a brief, masked word identical to the test item (e.g., Jacoby \& Whitehouse, 1989), and half were preceded by an unrelated masked word. Gallo et al. (2008) found that distinctive encoding reduced the fluency effect when participants were asked to respond both under time pressure and without any time constraints.

The results of Gallo et al. (2008) suggest that distinctive encoding may have reduced reliance on the fluency heuristic. This result is intriguing for two reasons. First, previous research on fluency attribution suggested that as long as there was some match between study and test (as was the case in Gallo et al.'s, 2008, study), participants would readily attribute the fluency from the prime as evidence of prior study (Westerman et al., 2002; Whittlesea et al., 1990). Second, reliance on fluency was usually 
greater when memory decisions were made under uncertainty, such as after an "artificial" study phase in which participants were told that study items were being presented when only visual or auditory static had been presented (Lloyd et al., 2003; Westerman et al., 2002; Westerman et al., 2003), or when the rememberer was less accurate (Verfaellie \& Cermak, 1999; Wolk, Schacter, Berman, Holcomb, Daffner and Budson, 2005). Given these data, a speeded test might be predicted to show enhanced priming effects in a manner similar to decisions made under uncertainty, because participants would not have time to closely evaluate the contents of memory. This may lead to a participant being more apt to rely on familiarity, which would be increased in the case of primed items.

The present experiments represent an attempt to examine the interaction between the fluency and distinctiveness heuristics in a variety of recognition test circumstances. Many models of recognition memory presume that both familiarity and recollection contribute to recognition memory decisions (Diana et al., 2006; Yonelinas, 2002). Thus, systematic study of the interplay of the decision heuristics that may underlie these variables will help to increase understanding of the roles that each of these processes play in a typical recognition memory decision.

\section{Experiment 1}

One recognition test format that might reveal new insight into the interaction between the fluency heuristic and the distinctiveness heuristic is the response reversal paradigm developed by Van Zandt and Maldonado-Molina (2004). In this test paradigm, participants are first asked to provide a speeded "yes" or "no" response to the test probe. This is followed by an opportunity to revisit the initial response and change the given response if desired. Examining the interaction between the fluency and distinctiveness heuristics within the response reversal paradigm is interesting for two reasons. The first of these is that the response reversal paradigm may help to further reveal the time course of the interaction between the fluency and distinctiveness heuristics. The experience of fluency is thought to be a rapid and automatic byproduct of speedy mental processing (Gallo et al., 2008; Whittlesea, 1993). In contrast, the distinctiveness heuristic relies on a slower and more effortful process of attempting to remember details, failing to do so, and then making attributions about why the recollection of details was not possible (Dodson \& Hege, 2005). Given these assumptions, it is reasonable to expect the fluency heuristic to act more quickly than the distinctiveness heuristic. Giving participants the opportunity to change their minds during a second recognition memory judgment should allow observation of the interplay between fluency and distinctiveness over the course of the test episode.
A second, related reason that the response reversal paradigm is of interest is that the knowledge that they will have two opportunities to respond to each test item may change the strategies that participants employ over the course of the test. Gallo et al., (2008) found that when participants were given fluent items and distinctive items during a speeded recognition test, participants reduced their reliance on fluency in the distinctive encoding condition. The researchers attributed this finding to the fact that distinctive encoding changed the subjective match between study and test, therefore leading participants to discount fluency even under pressure to respond quickly. If participants know that there are two opportunities to respond to each test stimulus, they may be less likely to rule out fluency as a source of evidence, because they know that fluency is valuable for making quick memory decisions and that they will have the opportunity to revise their answer later.

\section{Method}

\section{Participants}

A total of 126 undergraduate students from Seton Hall University participated in the experiment as partial fulfillment of a course requirement.

Materials and apparatus

The names of the objects that were to be presented in the picture group served as the word stimuli. The items were 3-12 letters in length and represented common names of objects (e.g., hammer, banana). Line drawings of the objects served as pictorial stimuli for half of the participants. The stimuli were identical to those used in a prior study (Westerman et al., 2003). All experiments were conducted on a desktop computer equipped with E-Prime software.

\section{Design}

The experiment had a 2 (encoding: distinctive or standard) by 2 (status: old or new) by 2 (prime type: matched or mismatched) by 2 (response: first or second) mixed-factor design. Encoding was manipulated between participants, and the other variables were manipulated within participants.

\section{Procedure}

Participants were tested individually in small testing rooms. They were told that they were partaking in a memory study but were not instructed regarding the specific nature of the test. They were then shown a list of either 66 words or the same words paired with line drawings. The first and last 3 study 
items served as buffers. Study and test items were presented in a random order for each participant. Each item appeared for $1 \mathrm{~s}$, followed by a 500 -ms blank screen. After the study phase, a 10-min distractor task (word search) was administered.

The recognition test was then given to the participants. The format of the memory test was the same for all participants and contained only words, regardless of the encoding condition. A total of 120 test items were presented. Half of these were old items representing the 60 items from the study phase, and half were new words that had not been presented during the study phase. Half of each class of items were preceded by a 34-ms masked prime that matched the test item, and the other half were preceded by a 34-ms masked prime that did not match the test word and did not appear during the study or test phases at any other time. Each prime was masked by a row of number signs

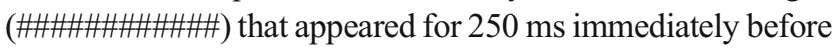
and after the prime. After the presentation of the masked prime, the screen was cleared for $800 \mathrm{~ms}$, and then the test item appeared. There were 30 words for each of four test categories (matched target, mismatched target, matched lure, mismatched lure). Across participants, the prime type (match or mismatch) and status (old or new) were counterbalanced, such that each item served in each of the four conditions approximately $1 / 4$ of the time.

Once the test item was presented, participants were instructed to make a fast "yes" or "no" response to the word (within $800 \mathrm{~ms}$ ). They were then given feedback about the speed of their response. A screen appeared that told them the response time on the previous response. Participants were instructed to attempt to speed up if their response time was longer than $800 \mathrm{~ms}$. The test item then appeared a second time, and participants again made a yes/no recognition decision. They were told that they could take as little or as much time as they wished to make this second decision and that they were welcome to change their response if they felt that the first judgment was wrong. Participants were told that the row of number signs would help them to focus attention and respond quickly. They were not told about the presence of the primes. Although this was a departure from the methods of Gallo et al. (2008), it was consistent with previous work on the fluency heuristic (Lloyd et al., 2003; Miller, Lloyd, \& Westerman, 2008; Westerman et al., 2002; Westerman et al., 2003). There were no intervening items between the first and second judgments of a word. Participation took approximately $25 \mathrm{~min}$

\section{Results and discussion}

As is customary in research using timed response pressure, all participant data were included in the analysis in order to avoid any item selection bias (e.g., Gallo, Weiss \&
Schacter, 2004). A significance criterion of .05 was used for all analyses. We present the overall ANOVAs for the first and second responses to look for differences in priming effects, which are interpreted as evidence of fluency effects, and then the follow-up planned contrasts to test for evidence of the distinctiveness heuristic. The means as a function of the response, encoding, status, and prime are presented in Fig. 1.

First response

A 2 (encoding: distinctive or standard) by 2 (status: old or new) by 2 (prime: match or mismatch) mixed-factor ANOVA was conducted using the proportion of "yes" responses as the dependent variable. There were main effects of status $\left[F(1,124)=426.83, \eta_{\mathrm{p}}^{2}=.78\right]$ and prime $\left[F(1,124)=56.92, \eta_{\mathrm{p}}^{2}=.32\right]$, because participants said "yes" more often to targets and matched items, respectively. There was no main effect of encoding, $F(1,124)=1.17$. Critically, there was no interaction between prime and encoding, $F(1,124)=2.92, p<.09$; the priming effects were similar for the word $(M=.09)$ and picture conditions
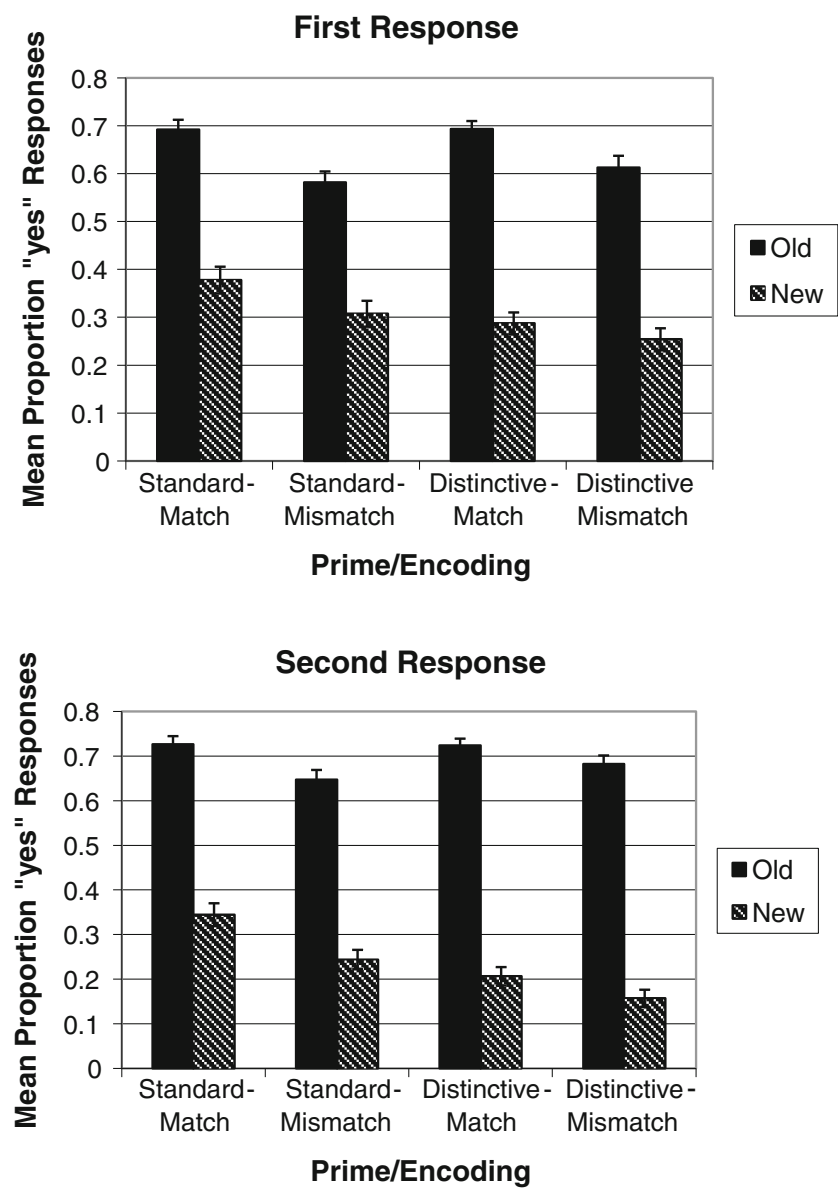

Fig. 1 Mean proportions of "yes" responses as a function of response, test item status, encoding condition, and prime for Experiment 1 
$(M=.06)$. There was a significant interaction between status and encoding, $F(1,124)=7.19, \eta_{\mathrm{p}}^{2}=.06$. Discrimination was better for pictures than for words. The interaction between status and prime was also significant, $F(1,124)=7.62, \eta_{\mathrm{p}}{ }^{2}=.06$. The priming effect was larger for targets $(M=.10)$ than for lures $(M=.05)$. The threeway interaction among prime, status, and encoding was not significant, $F<1$.

\section{Second response}

A 2 (encoding: distinctive or standard) by 2 (status: old or new) by 2 (prime: match or mismatch) mixed-factor ANOVA was conducted using the proportion of "yes" responses as the dependent variable. The results of this ANOVA were similar to those for the first decision, with two exceptions. Again, the main effects of status $[F(1,124)=$ $\left.831.13, \eta_{\mathrm{p}}{ }^{2}=.87\right]$ and prime $\left[F(1,124)=64.31, \eta_{\mathrm{p}}{ }^{2}=.34\right]$ were significant, due to the presence of more "yes" responses to targets and primed items, respectively. The main effect of encoding was also significant, $F(1,124)=5.06, \eta_{\mathrm{p}}{ }^{2}=.04$. Participants were more likely to say "yes" in the standard encoding condition $(M=.49)$ than in the distinctive encoding condition $(M=.44)$. However, unlike in the first decision, the interaction between encoding and prime was significant, $F(1$, $124)=7.03, \eta_{\mathrm{p}}{ }^{2}=.05$; participants who studied pictures showed a smaller priming effect $(M=.04)$ than did those who studied words $(M=.09)$. Follow-up tests showed that both priming effects were still significant $[t(62)=4.74, d=0.60$, and $t(62)=6.78, d=0.82$, for pictures and words, respectively]. In addition, the interaction between status and prime was no longer significant, because participants had similar priming effects for old $(M=.07)$ and new $(M=.07)$ items, $F(1,124)=1.05$. The interaction between status and encoding remained significant, due to better discrimination for participants who studied pictures than for those who only studied words, $F(1,124)=16.51, \eta_{\mathrm{p}}^{2}=.12$, Again, the threeway interaction was not significant, $F<1$.

\section{The distinctiveness heuristic}

The distinctiveness heuristic is evidenced by a reduced false alarm rate when a participant expects distinctive information (in this case, memory of a picture) to be diagnostic of prior study. We tested for an effect of study format on false alarms only in the mismatched prime condition, to be consistent with the methodology of Gallo et al. (2008), with the rationale that the matched prime condition would be affected by familiarity effects from the prime. There was no difference in the false alarm rates during the first decision, $t(126)=1.56, p<.14$. During the second response, fewer false alarms were made by participants who studied pictures, $t(126)=3.00$. This finding suggests that participants are more successful at reducing false alarms during nonspeeded recognition judgments (Dodson \& Hege, 2005; Gallo et al., 2008). Furthermore, the hit rates were equivalent in the two encoding conditions for both the first $[t(124)=0.58, p<.36]$ and second $[t(124)=0.67, p<.22]$ responses, suggesting that differences in accuracy are not sufficient to explain the advantage of studying pictures.

The results of Experiment 1 showed that participants in the picture condition performed similarly to those in the word condition during the speeded response portion of the memory test. Unlike in the results of Gallo et al. (2008), participants who studied pictures interpreted the fluency from the prime as evidence of prior study during a speeded response at a rate similar to those participants who studied only words. This finding is consistent with the ideas that the distinctiveness heuristic requires time to be used and that participants may respond differently when they are aware that they will have another opportunity to respond, allowing the opportunity for an answer to be adjusted. During the second response, participants in the picture condition showed evidence of applying both the fluency and distinctiveness heuristics to the recognition memory decision. This conclusion was evidenced by a significant priming effect for the picture group (fluency heuristic) as well as a reduced false alarm rate (distinctiveness heuristic). It is possible, however, that the finding of significant fluency effects during the second response may not be attributable to the prime. Because of the design of the study, participants were shown a prime, then made a recognition decision that was followed quickly by a second recognition decision. Given this methodology, it is possible that some perceptual priming was caused by the first test item. Of course, this concern is alleviated somewhat by the fact that the first test item always matched the second test item, regardless of prime condition, suggesting that the differences observed between the matching prime conditions and the mismatching prime conditions arose from the influence of the prime and not from the first test word. However, it is possible that the two tests may have interacted with the prime in some unforeseen way. Therefore, a second experiment was conducted to confirm that a JacobyWhitehouse style prime continues to influence memory decisions even when time has elapsed between the presentation of the prime and the final test item. In order to test whether fluency effects are still present after a lengthy delay between the prime and the test item, this experiment contained only one test response. In Experiment 2a, participants had to wait to give an answer to the test item. The delay was approximately the same as the average amount of time that elapsed between the prime and the second test response in Experiment 1. We ran a follow-up experiment to account for the fact that in the second response of the 
response reversal procedure, participants had more time to carefully reflect on their answers, weighing their confidence in their memory decisions. In Experiment $2 b$, participants were asked to rate their confidence on a scale from 1 to 4 , which also led to a delay in responding.

\section{Experiments 2a and 2b}

Experiments $2 \mathrm{a}$ and $2 \mathrm{~b}$ were designed to be identical to Experiment 1, with the exception that participants had only one chance to respond to each test stimulus. Once again, participants studied a list of words (standard encoding condition) or of words paired with a picture (distinctive encoding condition). During the recognition test, half of the test items were again primed to test for the fluency heuristic. However, participants had only one chance to respond to each test item. Critically, Experiment $2 \mathrm{a}$ was designed with timing parameters similar to those of Experiment 1, but without a first response. This led to a delay between the test word and the recognition decision. Experiment $2 b$ was identical to Experiment 2a, except that participants were asked to make their recognition decisions using a confidence scale rather than to wait to make a recognition decision. For each experiment, a comparison of the false alarm rates for pictures and words was used to assess the distinctiveness heuristic. Based on the results of Experiment 1, it was predicted that the results of Experiments $2 \mathrm{a}$ and $2 \mathrm{~b}$ would be similar to those observed in the second response phase of Experiment 1, with evidence for concurrent use of the distinctiveness and fluency heuristics in a recognition memory test.

\section{Method}

Participants

A total of 112 undergraduate students from Seton Hall University participated in Experiment 2a in partial fulfillment of a course requirement. A further 62 participants took part in Experiment 2b. In each experiment, half of the participants were assigned to the distinctive encoding condition (word + picture), and half were assigned to the standard encoding condition (word).

\section{Design}

Experiments $2 \mathrm{a}$ and $2 \mathrm{~b}$ both had 2 (encoding: standard or distinctive) by 2 (status of test word: old or new) by 2 (prime type: match or mismatch) mixed-factor designs. In each experiment, encoding was manipulated between participants, while prime and status were manipulated within participants.
Procedure

Experiment $2 a$ The experiment was identical to Experiment 1, with the following exceptions. First, participants made only one recognition response. Second, this response was made after the test word had appeared for $1,500 \mathrm{~ms}$ and was followed by a $100-\mathrm{ms}$ blank screen. This was done to approximate the average amount of time that elapsed between the first and second recognition decisions in Experiment 1.

Experiment $2 b$ The procedure was identical to Experiment 2a, with one exception. Rather than delaying the time between the presentation of the test word and a recognition decision, participants made their decisions on a 4-point confidence scale (sure old, unsure old, unsure new, sure new).

\section{Results and discussion}

\section{Experiment 2a}

The mean proportions of "yes" responses as a function of encoding condition, prime type, and test item status for Experiment 2a are presented in Fig. 2. These data were analyzed using a 2 (encoding: standard or distinctive) by 2 (status of test item: old or new) by 2 (prime type: match or mismatch) mixed-factor ANOVA. There was a main effect of status, $F(1,110)=730.06, \eta_{\mathrm{p}}{ }^{2}=.87$ : Participants said "yes" more often to targets $(M=.68)$ than to lures $(M=.21)$. There was also a main effect of prime, $F(1,110)=21.04, \eta_{\mathrm{p}}^{2}=.16$, in which participants said "yes" more frequently to test items preceded by a matching prime $(M=.47)$ than to those preceded by a mismatching prime $(M=.42)$. There was an interaction between encoding and status, $F(1,110)=8.99, \eta_{\mathrm{p}}{ }^{2}=.08$, in which participants showed better discrimination for distinctive encoding relative to standard encoding. The three-way

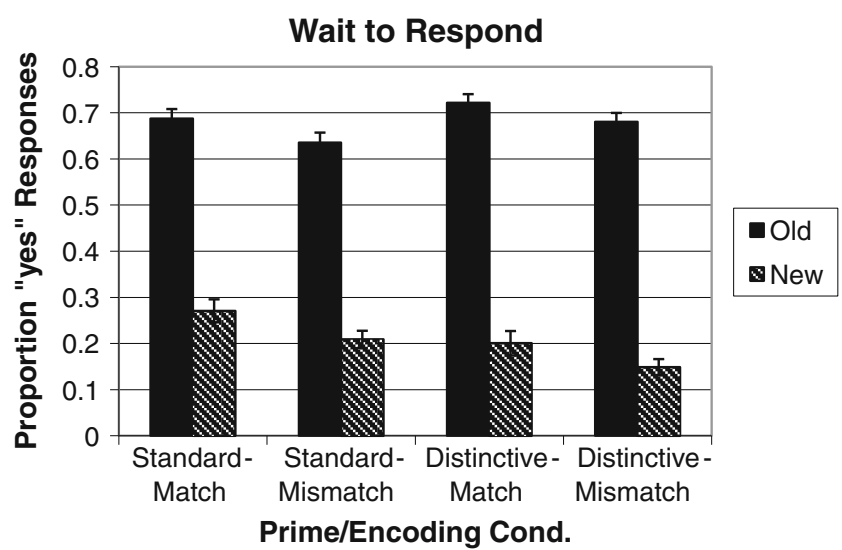

Fig. 2 Mean proportions of "yes" responses as a function of test item status, encoding, and prime for Experiment 2a 
interaction as well as the two-way interactions of prime with both test item status and encoding were not significant, $F$ s $<1$. This suggests that the magnitude of the priming effect was similar for participants in the distinctive $(M=.05)$ and standard $(M=.06)$ conditions. This is in contrast to the results of Gallo et al. (2008), who demonstrated that fluency effects were reduced following pictorial encoding.

Distinctiveness heuristic The distinctiveness heuristic is evidenced by a reduced false alarm rate for participants who studied words with pictures relative to studying only words. For items preceded by a mismatching prime, participants made fewer false alarms after picture study then after word study, $t(110)=2.53, d=0.44$, and the hit rates did not differ, $t(110)=1.55, p>.12$. Again, consistent with Gallo et al. (2008), studying pictures led to a reduced false alarm rate.

\section{Experiment $2 b$}

The complete results of Experiment $2 \mathrm{~b}$ are presented in Fig. 3. A 2 (encoding: distinctive or standard) by 2 (prime: match or mismatch) by 2 (status: old or new) mixed-factor ANOVA was conducted using the proportions of "old" responses as the dependent variable. A separate analysis using confidence level as an independent variable showed only that participants used the "sure old" response to a greater degree for old items than for new items. This finding is akin to a standard old/new effect, in which more "yes" responses are given to old than to new items.

The main effect of status was significant, $F(1,60)=$ $537.27, \eta_{\mathrm{p}}{ }^{2}=.90$; participants said "old" more often to old items $(M=.70)$ than to new items $(M=.25)$. The main effect of encoding was also significant, $F(1,60)=3.96, \eta_{\mathrm{p}}{ }^{2}=.06$; participants responded "old" more often in the word condition $(M=.49)$ than in the picture condition $(M=.45)$. The main effect of prime was significant, $F(1,60)=19.78, \eta_{\mathrm{p}}{ }^{2}=.25$, in that participants were more likely to use "old" responses for matched items $(M=.50)$ than for mismatched items $(M=.45)$. The interaction between status and encoding was marginally significant, $F(1,60)=3.75, p<.06, \eta_{\mathrm{p}}{ }^{2}=.06$. Although the hit rates were similar for the word and picture groups, there was a reduced false alarm rate for the picture group. There were no significant interactions of prime with status, $F(1,60)=1.31, p<.26$, or with encoding, $F(1,60)=$ 2.00, $p<.17$. As in Experiment 2a, the priming effect was not attenuated for words relative to pictures. The three-way interaction was also not significant, $F<1$.

Distinctiveness heuristic In order to test whether participants benefited from picture encoding through a reduced false alarm rate, independent-samples $t$-tests were conducted to compare the false alarm rates in the picture and word groups. There was a marginally significant lower false alarm rate for the mismatched new items $(M=.25)$ in the picture group relative to the word group $(M=.19), t(60)=$ $1.83, p<.07$ two-tailed. The effect for matched new items was significant, as participants had a lower false alarm rate after picture study $(M=.23)$ than after word study $(M=.33)$, $t(60)=2.78, d=0.60$. There were no differences in hit rates as a function of encoding for either matched items or mismatched items, $t \mathrm{~s}<1, p \mathrm{~s}<.56$.

When viewed in conjunction with the results of Experiment 1, the results of Experiments $2 \mathrm{a}$ and $2 \mathrm{~b}$ converge to suggest that participants can effectively apply both the fluency and distinctiveness heuristics over the course of a single test episode. Similar priming effects for items preceded by a matching prime were found independent of encoding condition. However, the picture groups had reduced false alarm rates relative to the word groups. Unlike Gallo et al. (2008) and the second decision of our Experiment 1, Experiments $2 \mathrm{a}$ and $2 \mathrm{~b}$ did not show an attenuation of the priming effect for the picture group relative to the word group.

Generally, the present results suggest that distinctive encoding did not always lead participants to reduce their
Fig. 3 Mean proportions of "old" responses as a function of confidence level, encoding, prime, and test item status for Experiment 2b. Dark bars are "sure" responses, and light bars are "unsure" responses

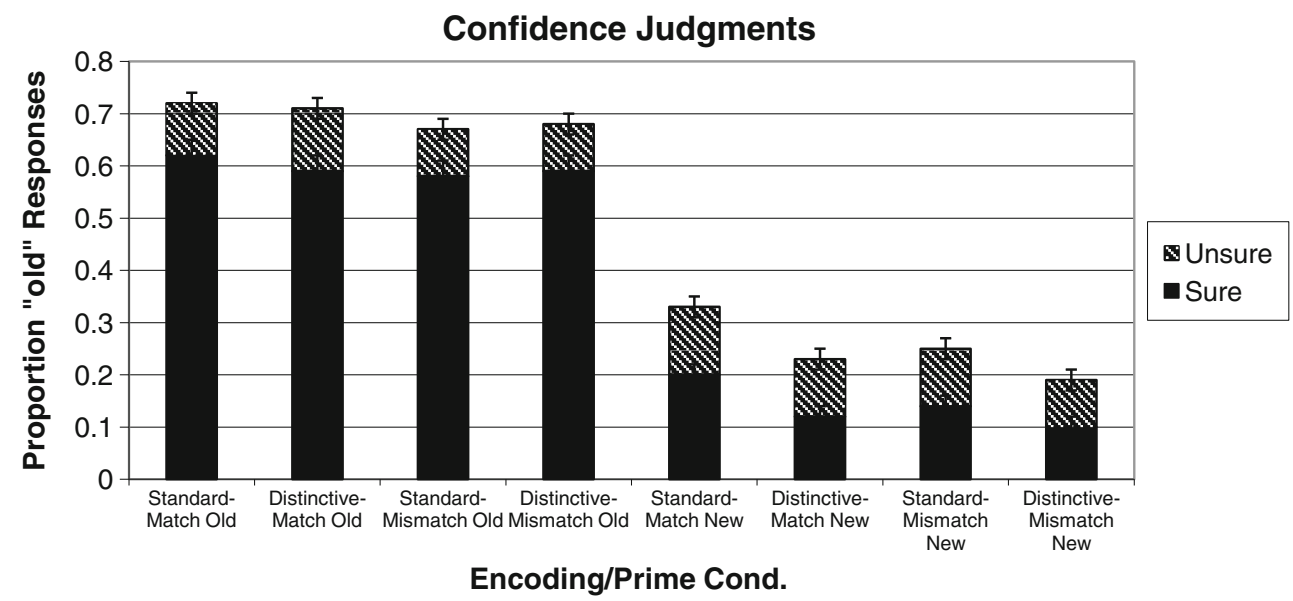


reliance on fluency as a cue to memory. In particular, they suggest that participants considered the properties of the retrieval episode as they prioritized the sources of evidence available to them. Taking more time to make a memory decision or making more nuanced memory decisions such as confidence judgments seems to have encouraged participants to adopt a balanced response strategy in which fluency and distinctiveness were each adopted as valid sources of evidence. Furthermore, these results suggest that the fluency heuristic continued to play an important role in recognition decisions, even when such powerful cues as distinctiveness were available.

\section{Experiment 3}

Manipulations of fluency are not limited to those with a matching prime. Research has demonstrated that increased conceptual fluency can also lead to increased "yes" responses on a recognition test. For example, participants are more likely to endorse a test item (e.g., ВОАТ) as having been studied if a highly predictive sentence stem is presented (e.g., "The stormy seas tossed the __.") relative to a stem for which many potential items would be appropriate (e.g., "She saved her money and bought a .") (see, e.g., Miller et al., 2008; Whittlesea, 1993; Whittlesea \& Williams, 2001). Again, it is assumed that this fluency from the sentence stem is interpreted as enhanced familiarity during the recognition test. The goal of Experiment 3 was to explore the interaction between the distinctiveness and fluency heuristics when the conceptual fluency of the test stimulus was explicitly manipulated. (Although the Jacoby-Whitehouse technique is often described as influencing perceptual fluency, there is also some conceptual fluency from the primes.) It is difficult to predict whether distinctive encoding will reduce fluency effects after a conceptual fluency manipulation. On one hand, some research has argued that fluency is experienced generically (Masson \& Caldwell, 1998), suggesting that conceptual and perceptual forms of fluency should be attributed in similar fashions. On the other hand, conceptual illusions are observed when perceptual fluency effects are not, such as after auditory study (Miller et al., 2008; Thapar \& Westerman, 2009).

\section{Method}

Participants

A total of 132 students from Seton Hall University participated in partial fulfillment of a course requirement.

\section{Materials}

The words and pictures were changed in Experiment 3 in order to use the predictive sentence stems from Kurilla and Westerman (2008) and Whittlesea (1993). Thirty critical words were selected along with six filler words that were not part of the test phases.

\section{Procedure}

Participants were tested individually on computers equipped with E-Prime 2.0. During the study phase, a list of 36 items ( 30 critical and 6 primacy and recency buffers) were presented one at a time. Participants in the distinctive encoding condition received each word paired with a picture, similar to Experiments 1, 2a, and 2b. The pictures were all selected from Microsoft Office's (Microsoft, Inc., Redmond, WA) clip art files and were chosen to be of similar levels of detail and size. After the study phase, participants worked on a distractor task for $10 \mathrm{~min}$. During the test phase, each test word was preceded by a sentence stem. Half of these sentences were predictive of the test word. For example, participants in the predictive stem condition might be given the sentence stem "The stormy seas tossed the ," which would be paired with the test word BOAT. In the nonpredictive sentence stem condition, participants might be given the sentence stem "She saved her money and bought a __ , to be paired with the test word BOAT (Whittlesea \& Williams, 2001). In this case, the word made sense, but so would have many other test words. Participants' reading of the sentence stem was self-paced, and they pressed a space bar once they had completed reading the stem. The test word then appeared on the next screen. Participants were instructed to use the "Y" key if they believed that the word was from the study list and "N" key if they thought that the word was not on the study list. In total, 60 items appeared on the test list. Half were targets and half were lures. Of these items, half were preceded by a predictive sentence stem, and half by a nonpredictive stem. Old or new status as well as sentence type was counterbalanced across participants. The order of the study and test items was randomly selected for each participant.

\section{Results and discussion}

A 2 (encoding: distinctive or standard) by 2 (status: old or new) by 2 (sentence context: predictive or nonpredictive) mixed-factor ANOVA was conducted using the proportion of "yes" responses as the dependent variable. Figure 4 presents the means as a function of the independent variables. The results were straightforward. There was a 


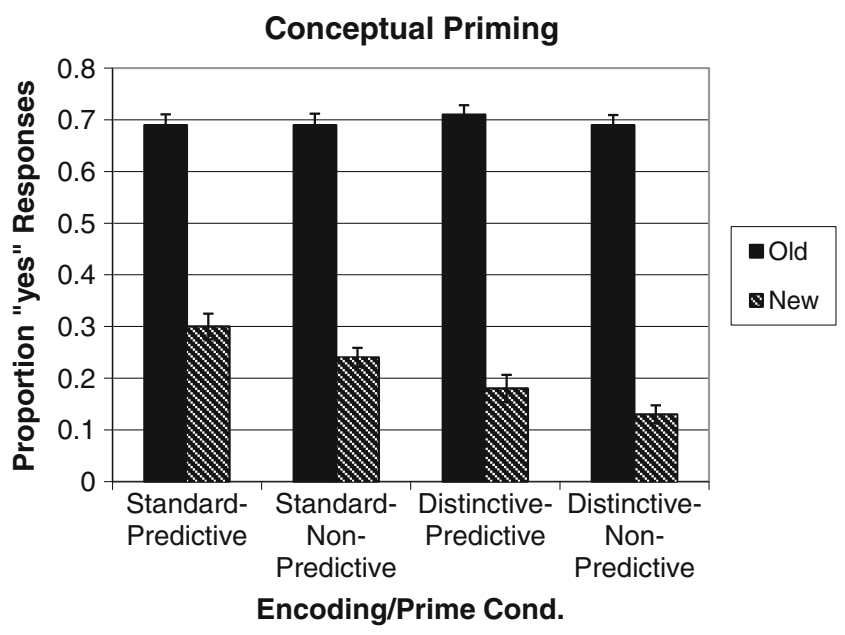

Fig. 4 Mean proportions of "yes" responses as a function of test item status, encoding, and sentence context for Experiment 3

main effect of status, $F(1,130)=641.57, \eta_{\mathrm{p}}{ }^{2}=.83$ : Participants recognized more old items $(M=.70)$ than new items $(M=.22)$. There was also a main effect of prime, $F(1,130)=10.26, \eta_{\mathrm{p}}{ }^{2}=.07$, in that participants recognized more predictive items $(M=.47)$ than nonpredictive items $(M=.44)$. This was qualified by a status by prime interaction, $F(1,130)=4.71, \eta_{\mathrm{p}}^{2}=.04$; as may be noted in Fig. 4, the priming effect was driven by the lure condition. Critically, the prime by encoding interaction was not significant, $F<1$. The priming effects were of similar magnitudes for the two encoding groups. There was a main effect of encoding, $F(1,130)=7.72, \eta_{\mathrm{p}}{ }^{2}=.06$, in which participants said "yes" more often in the word condition $(M=.48)$ than in the picture condition $(M=.43)$. This was qualified by an interaction between encoding and status, $F(1,130)=10.95, \eta_{\mathrm{p}}{ }^{2}=.08$. Participants had similar hit rates in both encoding conditions $(t \mathrm{~s}<1)$, but different false alarm rates, as is typical in experiments on the distinctiveness heuristic. Again, false alarm rates for the picture and word groups differed in the nonpredictive (analogous to mismatched) condition, $t(130)=3.89, d=0.55$. The three-way interaction was not significant, $F<1$.

The results of Experiment 3 show that participants continued to employ both the fluency and distinctiveness heuristics when conceptual fluency was manipulated rather than perceptual fluency. These results suggest that the fluency heuristic and the distinctiveness heuristic interact similarly, regardless of the type of fluency that participants are exposed to: Both perceptual and conceptual fluency were judged to be valuable sources of evidence, even in the presence of powerful cues to memory such as distinctiveness. In addition, as had also been observed with delayed responses and confidence judgments, fluency was used to a similar degree for participants in the word and picture conditions. These findings are consistent with those of
Experiments $2 \mathrm{a}$ and $2 \mathrm{~b}$ in demonstrating that participants did not always temper their reliance on fluency when study list items were distinctively encoded.

\section{General discussion}

The results of the present experiments suggest that participants are capable of engaging two response heuristics over the course of a single recognition decision: fluency and distinctiveness. However, the heuristics manifested themselves in different ways and on different time courses across the span of the recognition test episode. In Experiment 1, evidence suggesting that participants used the fluency heuristic (as measured by priming effects) was present in both the picture and word groups during both the initial and second responses of the recognition test. The distinctiveness heuristic, as measured by a decreased false alarm rate to items after pictorial encoding, was only evident during the second response. In Experiment 2a, all participants were forced to wait to make a memory decision. Again, the use of multiple heuristics was demonstrated by the pictorial encoding group, because their false alarm rate was lower than that of the word groupdemonstrating the distinctiveness heuristic - and participants also attributed the fluency from the prime as evidence that an item had been studied - thus demonstrating the fluency heuristic. A similar pattern of results was observed in Experiment $2 b$, when participants made a confidence judgment. Finally, in Experiment 3, participants attributed the conceptual fluency from a sentence stem in similar ways, regardless of whether they had studied only words or words paired with pictures. Generally, the results of these experiments support the idea that participants engage both the fluency and distinctiveness heuristics concurrently across the course of a recognition test episode.

The experiments reported here follow those of Gallo et al. (2008) in attempting to answer the question of how fluency attribution strategies interact with other memory strategies such as the distinctiveness heuristic. In contrast to the present study, Gallo et al. (2008) found that distinctive encoding reduced the magnitude of fluency effects from a masked prime. In the present study, consistent priming effects were observed for both distinctively and nondistinctively encoded study items across a variety of test circumstances, with the exception of the second response in Experiment 1. These results demonstrated that distinctive encoding does not always induce participants to discount fluency. However, due to the methodological differences between the present experiments and those of Gallo et al. (2008), further empirical work will be necessary in order to determine the circumstances under which participants are likely to discount fluency following exposure to distinctive 
encoding. Generally, the present results are consistent with the mismatch hypothesis proposed by Westerman et al. (2002) to account for their participants' use of fluency as a source of evidence for their memory decisions whenever there was a perceptual match between the study and test episodes.

One constant across the present experiments and those presented by Gallo et al. (2008) is that, despite the array of different circumstances presented across each of the experiments, participants consistently relied on distinctiveness as a cue to memory. The consistency with which participants applied the distinctiveness heuristic might lead some to speculate that they prioritized distinctiveness as a preferred cue to oldness and applied it as evidence of previous experience whenever the retrieval circumstances allowed for effective retrieval of distinctiveness cues. Indeed, the present results provide some support for this idea, in that distinctiveness effects were observed across all experiments. However, the present experiments also found little evidence suggesting that participants' reliance on distinctiveness as a cue to memory consistently overshadowed other types of evidence that may have been available at the time of test. In one view, it might be seen as surprising that participants continue to weigh fluency into their memory decisions in a test context in which fluency has been experimentally manipulated to be a rather unreliable cue to memory, especially given the fact that during the same test episode distinctiveness remained a consistent and reliable indicator of oldness. However, this result is less surprising in light of the fact that outside of the Jacoby-Whitehouse paradigm, fluency is an enormously reliable indicator of recent previous experience with a stimulus. The present results provide preliminary evidence suggesting that memory systems optimize performance by drawing on all relevant information streams, capably engaging multiple heuristics in order to arrive at a decision. These findings suggest that (at least under the present experimental conditions) two heuristics are better than one. Across experiments, distinctive encoding did not change the hit rate, but did reduce false alarm rates. Coupled with the increased hit rates for old items that are primed, this combination of heuristic use leads to an overall increase in accuracy.

The present results also expanded our knowledge of how the fluency and distinctiveness heuristics interact by manipulating the type of fluency participants were exposed to at test. The results of Experiment 3 suggested that the fluency heuristic and the distinctiveness heuristic functioned similarly, regardless of whether the fluency manipulation was primarily perceptual or conceptual. Participants factored the conceptual fluency of the test item into their memory decisions, even when the item had been distinctively encoded. These results further support the claim that memory systems are capable of integrating and accounting for multiple forms of evidence as they make their memory judgments.

The present results are generally compatible with dualprocess models of memory (for reviews, see Diana et al., 2006; Yonelinas, 2002). Fluency is thought to be a component of familiarity, and the attribution of fluency is governed by the fluency heuristic. Participants interpreted fluency as evidence of previous occurrence during both speeded and nonspeeded recognition responses. Recollection, which is associated with slower, effortful, detail-driven processing, is the central mechanism by which the distinctiveness heuristic is theorized to operate (Gallo, 2004). Consistent with this conceptualization, the distinctiveness heuristic was only evidenced during the nonspeeded recognition judgments. The finding that participants used both fluency and distinctiveness during a recognition decision is perhaps most consistent with dualprocess models of memory that assume that both familiarity and recollection influence an individual memory decision (e.g., Wixted, 2007). A second way to conceptualize the results of the present study is an approach to recognition memory in which responses are a function of the participants' expectations and the demands of the test (Malmberg, 2008). In each of the experiments reported here, participants were able to effectively monitor the sources of information available to them and tailored their response strategy to the demands of the test.

Overall, the experiments presented here increase our knowledge of the factors that participants weigh as they employ various decision heuristics when they are about to make a memory decision. Generally, the results showed that participants effectively applied multiple memory heuristics over the course of a recognition test episode, optimizing their response strategies by weighing the match between the study and test conditions, the types and quality of evidence available to them at the time of test, and the demands of the test situation. Although this article has focused on the distinctiveness heuristic and the fluency heuristic and demonstrated that participants willingly employed each of these over the course of a memory decision, it is important to remember that there are more memory heuristics that participants may choose to employ as they make their memory decisions. Future research may find it productive to further map the interactions between other known response heuristics (e.g., the "take the best" heuristic; Gigerenzer \& Goldstein, 1996). It is important to understand how people react to complex memory decisions where multiple sources of evidence and multiple response strategies are available to them, because these circumstances mirror the complex real-world memory decisions made numerous times a day. We believe that an approach to the study of memory that focuses on mapping the interactions between the various response strategies people can employ will eventually lead to 
a fuller understanding of how human memory operates outside the carefully controlled laboratory environment.

\section{References}

Bernstein, I. H., \& Welch, K. R. (1991). Awareness, false recognition, and the Jacoby-Whitehouse effect. Journal of Experimental Psychology. General, 120, 324-328.

Clarke, R., \& Morton, J. (1983). Cross-modality facilitation in tachistoscopic word recognition. The Quarterly Journal of Experimental Psychology, 35A, 79-96.

Diana, R. A., Reder, L. M., Arndt, J., \& Park, H. (2006). Models of recognition: A review of arguments in favor of a dual-process account. Psychonomic Bulletin \& Review, 13, 1-21.

Dodson, C. S., \& Hege, A. C. G. (2005). Speeded retrieval abolishes the false-memory suppression effect: Evidence for the distinctiveness heuristic. Psychonomic Bulletin \& Review, 12, 726-731.

Dodson, C. S., \& Schacter, D. L. (2002). When false recognition meets metacognition: The distinctiveness heuristic. Journal of Memory and Language, 46, 782-803.

Gallo, D. A. (2004). Using recall to reduce false recognition: Diagnostic and disqualifying monitoring. Journal of Experimental Psychology. Learning, Memory, and Cognition, 30, 120-128.

Gallo, D. A., Perlmutter, D. H., Moore, C. D., \& Schacter, D. L. (2008). Distinctive encoding reduces the Jacoby-Whitehouse illusion. Memory \& Cognition, 36, 461-466.

Gallo, D. A., Weiss, J. A., \& Schacter, D. L. (2004). Reducing false recognition with criteria recollection tests: Distinctiveness heuristic versus criterion shifts. Journal of Memory and Language, 51, 473 493.

Gigerenzer, G., \& Goldstein, D. G. (1996). Reasoning the fast and frugal way: Models of bounded rationality. Psychological Review, 103, 650-669.

Jacoby, L. L., \& Dallas, M. (1981). On the relationship between autobiographical memory and perceptual learning. Journal of Experimental Psychology. General, 110, 306-340.

Jacoby, L. L., \& Whitehouse, K. (1989). An illusion of memory: False recognition influenced by unconscious perception. Journal of Experimental Psychology. General, 118, 126-135.

Kurilla, B. P., \& Westerman, D. L. (2008). Processing fluency affects subjective claims of recollection. Memory \& Cognition, 36, 82-92.

Lloyd, M. E., Westerman, D. L., \& Miller, J. K. (2003). The fluency heuristic in recognition memory: The effect of repetition. Journal of Memory and Language, 48, 603-614.

Malmberg, K. J. (2008). Recognition memory: A review of the critical findings and an integrated theory for relating them. Cognitive Psychology, 57, 335-384.

Masson, M. E. J., \& Caldwell, J. I. (1998). Conceptually driven encoding episodes create perceptual misattributions. Acta Psychologica, 98, 183-210.
Miller, J. K., Lloyd, M. E., \& Westerman, D. L. (2008). When does modality matter? Perceptual versus conceptual fluency-based illusions in recognition memory. Journal of Memory and Language, 58, 1080-1094.

Murrell, G. A., \& Morton, J. (1974). Word recognition and morphemic structure. Journal of Experimental Psychology, 102, 963-968.

Neisser, U. (1954). An experimental distinction between perceptual process and verbal response. Journal of Experimental Psychology, 47, 399-402

Schacter, D. L., Israel, L., \& Racine, C. (1999). Suppressing false recognition in younger and older adults: The distinctiveness heuristic. Journal of Memory and Language, 40, 1-24.

Thapar, A., \& Westerman, D. L. (2009). Aging and fluency-based illusions in recognition memory. Psychology and Aging, 24, 595-603.

Van Zandt, T., \& Maldonado-Molina, M. M. (2004). Response reversals in recognition memory. Journal of Experimental Psychology. Learning, Memory, and Cognition, 30, 1147-1166.

Verfaellie, M., \& Cermak, L. S. (1999). Perceptual fluency as a cue for recognition judgments in amnesia. Neuropsychology, 13, 198205. doi:10.1037/0894-4105.13.2.198.

Westerman, D. L., Lloyd, M. E., \& Miller, J. K. (2002). On the attribution of perceptual fluency in recognition memory: The role of expectation. Journal of Memory and Language, 47, 607-617.

Westerman, D. L., Miller, J. K., \& Lloyd, M. E. (2003). Change in perceptual form attenuates the use of the fluency heuristic in recognition. Memory \& Cognition, 31, 619-629.

Westerman, D. L. (2008). Relative fluency and illusions of recognition memory. Psychonomic Bulletin \& Review, 15, 1196-1200.

Whittlesea, B. W. A. (1993). Illusions of familiarity. Journal of Experimental Psychology. Learning, Memory, and Cognition, 19, $1235-1253$.

Whittlesea, B. W. A., Jacoby, L. L., \& Girard, K. (1990). Illusions of immediate memory: Evidence of an attributional basis for feelings of familiarity and perceptual quality. Journal of Memory and Language, 29, 716-732.

Whittlesea, B. W. A., \& Williams, L. D. (2001). The discrepancyattribution hypothesis II: Expectation, uncertainty, surprise, and feelings of familiarity. Journal of Experimental Psychology. Learning, Memory, and Cognition, 27, 14-33.

Wixted, J. T. (2007). Dual-process theory and signal-detection theory of recognition memory. Psychological Review, 114, 152-176.

Wolk, D. A., Schacter, D. L., Berman, A. R., Holcomb, P. J., Daffner, K. R., \& Budson, A. E. (2005). Patients with mild Alzheimer's disease attribute conceptual fluency to prior experience. Neuropsychologia, 43, 1662-1672.

Yonelinas, A. P. (1999). The contribution of recollection and familiarity to recognition and source memory judgments: A formal dual process model and an analysis of receiver operating characteristics. Journal of Experimental Psychology. Learning, Memory, and Cognition, 25, 1415-1434.

Yonelinas, A. P. (2002). The nature of recollection and familiarity: A review of 30 years of research. Journal of Memory and Language, 46, 441-517. 